\title{
Retracted: Similarities between Initiation Mechanism between Cotton Fiber and Arabidopsis Trichome: Prospects in Improving Cotton Fiber Yield
}

\author{
Anh Phu Nam Bui ${ }^{1,2^{*}}$, Binh-Nguyen Ong ${ }^{3}$, Thuan-Anh Nguyen Huu ${ }^{3}$ \\ and Hoang-Dung $\operatorname{Tran}^{3^{*}}$ \\ ${ }^{1}$ Faculty of Biotechnology, Ho Chi Minh city Open University, 35 Ho Hao Hon street, \\ Ho Chi Minh city, Vietnam. \\ ${ }^{2}$ Department of Cell Biology, Texas Tech University Health Sciences Center, 3601 th Street, \\ Lubbock, Texas 79430, USA. \\ ${ }^{3}$ Faculty of Biotechnology, Nguyen Tat Thanh University, 298A-300A Nguyen Tat Thanh Street, \\ Ward 13, District 4, Ho Chi Minh 72820, Vietnam.
}

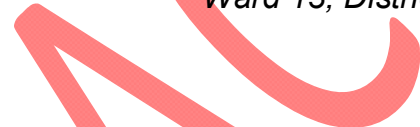

Authors' contributions

This work was carried out in collaboration among all authors. Author APNB designed the study and wrote the first draft of the manuscript. Authors BNO and TANH managed the analyses of the study. Author HDT managed the literature searches and edited the final manuscript. All authors read and

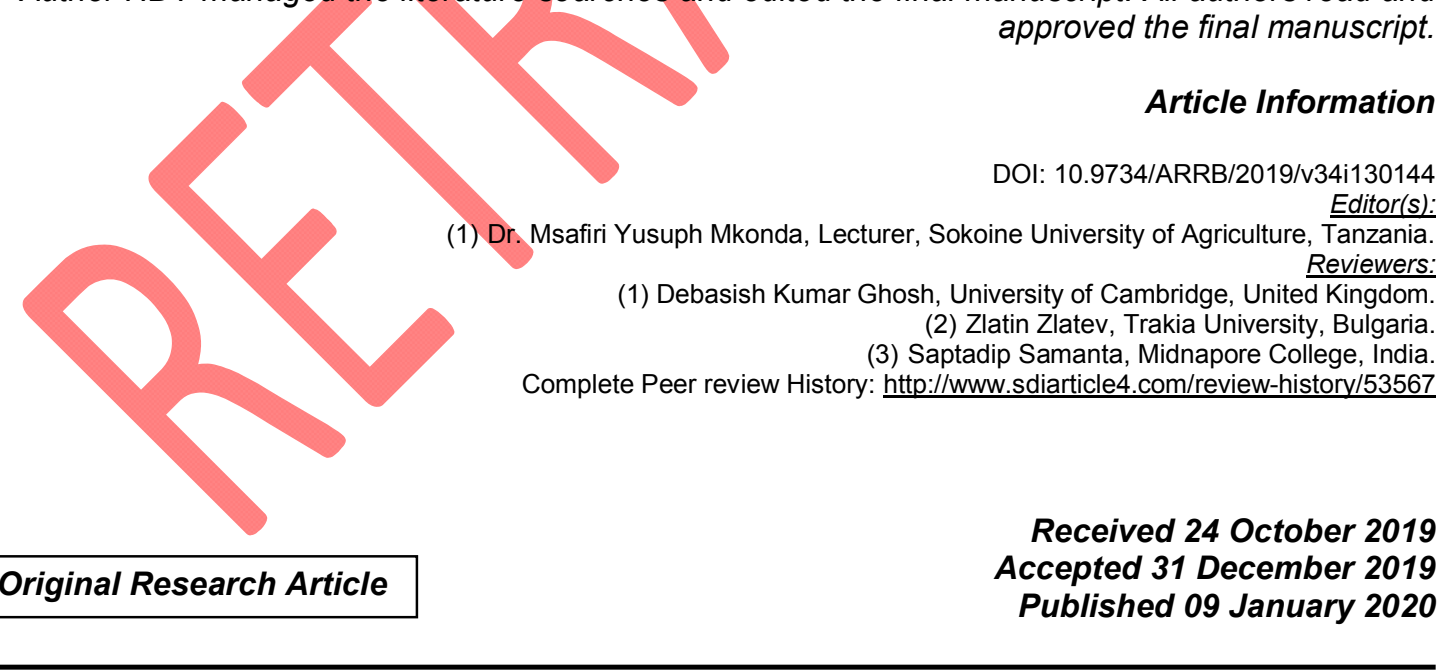

ABSTRACT

Cotton fiber is the fundamental material for a textile industry, and currently there is an immense interest in understanding the process of fiber initiation and development. Cotton fiber, also known as seed trichome, is differentiated from the seed coat epidermal cells similar to Arabidopsis leaf trichome, which is differentiated from the leaf epidermal cells. Despite functional characterization of individual cotton fiber initiation genes, currently there is not a comprehensive understanding of the 
mechanism behind cotton fiber initiation. Since the resemblance in initiation to cotton fiber, the Arabidopsis trichome has been successfully employed as a model system for functional characterization of cotton fiber initiation genes. Knowledge gained from the initiation mechanism of Arabidopsis trichomes will facilitate, as a comparative model system in understanding of the cotton fiber initiation mechanisms.

Keywords: Trichome; fiber initiation; trimeric complex; diploid; tetraploid

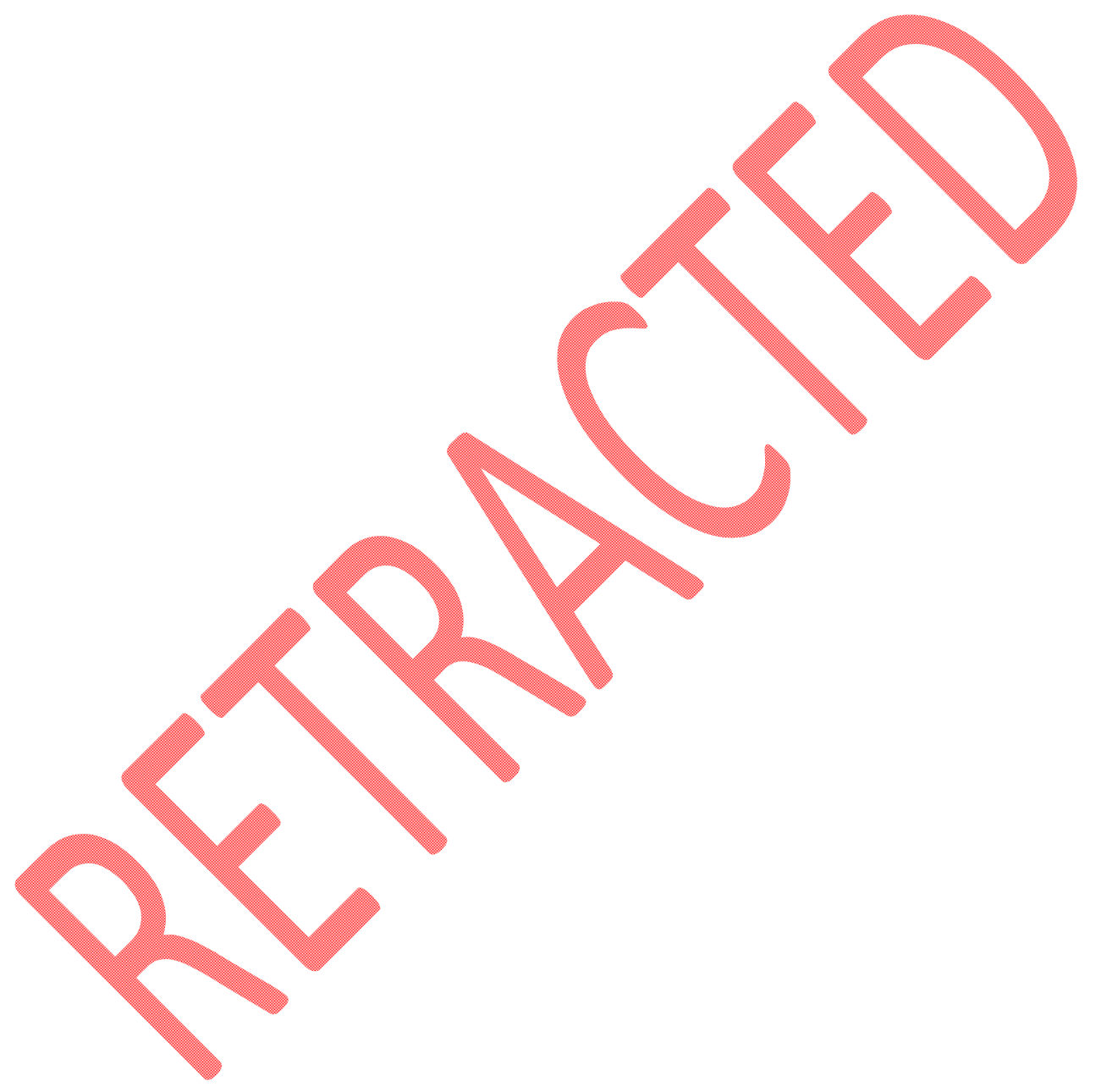

